\title{
THE EFFECT OF SMOOTHNESS ON VARIATION
}

\author{
P. G. HOWLETT
}

(Received 6 April 1972)

Communicated by J. Virsik

\section{Introduction}

Let $R$ be the set of real numbers, and let $S_{1}$ denote the class of all real valued functions $f$ on $R$ which are smooth to the first order (i.e. the derivative $f^{(1)}$ exists and is continuous) and have compact support. The first order variation of $f$ on an open set $U$ is given by

$$
I_{1}(f, U)=\int_{U}\left|f^{(1)}(x)\right| d x
$$

and in the case where $U=R$ we have the total first order variation of $f$, usually denoted by $I_{1}(f)$.

$$
I_{1}(f)=\int\left|f^{(1)}(x)\right| d x
$$

We wish to establish an alternative expression for the total first order variation. Let $P$ be the set on which $f^{(1)}$ is non zero. Since $P$ is open we can find a countable collection of mutually disjoint open intervals $\left(a_{1 j}, b_{1 j}\right)$ such that

$$
P=\bigcup_{j=1}^{\infty}\left(a_{1 j}, b_{1 j}\right)
$$

Now we define $d_{j}=\left|f\left(b_{1 j}\right)-f\left(a_{1 j}\right)\right|>0$ and hence $d_{j}$ is the first order variation of $f$ on the interval $\left(a_{1 j}, b_{1 j}\right)$. Thus it is easily seen that

$$
I_{1}(f)=\sum_{j=1}^{\infty} d_{j}
$$

We will show that the sequence $\left\{d_{j}\right\}$ can be rearranged to give a sequence $\left\{d_{j_{n}}\right\}$ such that $d_{j_{n}} \geqq d_{j_{n+1}}$ for all $n=1,2, \cdots$ and such that

$$
d_{j_{n}}=\frac{c_{n}}{n} \text { all } n=1,2, \cdots
$$


where $c_{n} \rightarrow 0$ as $n \rightarrow \infty$. If, for each $\lambda>0$ we define the open set $F_{\lambda}=\{x \mid x \in R$ and $0<f(x)<\lambda\}$ then it is possible to show that

$$
\lim _{\lambda \rightarrow 0} I_{1}\left(f, F_{\lambda}\right)=0 \text {. }
$$

Let $m$ be a natural number. We use $S_{m+1}$ to denote the subclass of $S_{1}$ containing all functions $f$ which are smooth to order $m+1$ (i.e. the $(m+1)$ th derivative $f^{(m+1)}$ exists and is continuous). It seems intuitively reasonable that increased smoothness in $f$ will be associated with decreased first order variation. We will show that it is now possible to write

$$
d_{j_{n}}=\frac{k_{n}}{n^{m+1}} \text { all } n=1,2, \cdots
$$

where $k_{n} \rightarrow 0$ as $n \rightarrow \infty$ and hence to show that

$$
\lim _{\lambda \rightarrow 0} \lambda^{1 /(m+1)-1} \cdot I_{1}\left(f, F_{\lambda}\right)=0 .
$$

It is now possible to explain the origin of the problem and to suggest an extension and application of the above results. Let $f \in L$ (the class of all integrable functions). The first order variation of $f$ on an open set $U$ can be defined as

$$
I_{1}(f, U)=\sup _{\psi} \int f(x) \psi^{(1)}(x) d x
$$

where the supremum is taken over all infinitely differentiable functions $\psi$ such that spt. $(\psi) \subset U$ and with $\|\psi\| \leqq 1$ (we are using the uniform norm). This definition can now be extended to cover more general sets. When $U=R$ we have the total first order variation $I_{1}(f)$. We use $B_{1}$ to denote the subclass of $L$ consisting of those $f$ with compact support for which $I_{1}(f)$ is finite. We can now use the co-area formula $([1])$ to show that

$$
\lim _{\lambda \rightarrow 0} I_{1}\left(f, F_{\lambda}\right)=0
$$

Following investigations by Michael ([5], [6], [7], ) and Goffman ([2], [3]) which make implicit use of this result it is possible to state the following theorem

"Let $f \in B_{1}$ and choose $\varepsilon>0$. We can find $g \in S_{1}$ such that the set $\{x \mid x \in R$ and $f(x) \neq g(x)\}$ has measure less than $\varepsilon$ and such that $I_{1}(g)<I_{1}(f)+\varepsilon$."

Let $m$ be a natural number. The $(m+1)$ th order variation of $f$ on an open set $U$ can be defined as 


$$
I_{m+1}(f, U)=\sup _{\psi} \int f(x) \psi^{(m+1)}(x) d x
$$

where the supremum is taken over all infinitely differentiable functions $\psi$ such that $\operatorname{spt}(\psi) \subset U$ and with $\|\psi\| \leqq 1$. This definition can now be extended to cover more general sets. When $U=R$ we obtain the total $(m+1)$ th order variation. Let $B_{m+1}$ denote the subclass of $B_{1}$ for which $I_{m+1}(f)$ is finite. For $m=1$, the method described above for smooth functions can be modified ([4]) to show that

$$
\lim _{\lambda \rightarrow 0} \lambda^{1 /(m+1)-1} I_{1}\left(f, F_{\lambda}\right)=0
$$

This result is then used to establish the theorem stated below

"Let $f \in B_{m+1}$ and choose $\varepsilon>0$. We can find $g \in S_{m+1}$ such that the set $\{x \mid x \in R$ and $f(x) \neq g(x)\}$ has measure less than $\varepsilon$ and such that $I_{m+1}(g)<I_{m+1}(f)+\varepsilon$."

It is believed that this theorem can be proved for all values of $m$ and that the work in this paper can be modified to provide a basis for the proof. It should be noted however that, as in [4], these result may only provide a partial answer to the corresponding theorem in $R^{2}$.

\section{Variation in $S_{1}$}

Let $f \in S_{1}$. Since $f^{(1)}$ is continuous and has compact support it follows from the integral definition that

$$
I_{1}(f)<\infty
$$

LEMMA 2.1. The sequence $\left\{d_{j}\right\}$ can be rearranged to a sequence $\left\{d_{j_{n}}\right\}$ such that $d_{j_{n}} \geqq d_{j_{n}+1}$ for all $n=1,2, \cdots$ and in fact we can write

$$
d_{j_{n}}=\frac{c_{n}}{n} \text { all } n=1,2, \cdots
$$

where $\left\{c_{n}\right\}$ is bounded and has limit zero.

ProOf. Let $D=\left\{d_{j}\right.$ for all $\left.j=1,2, \cdots\right\}$

$$
D_{r}=\left\{d_{j} \mid \frac{1}{r-1}>d_{j} \geqq \frac{1}{r}\right\} \text { each } r=1,2,3, \cdots
$$

Since $d_{j}>0$ all $j=1,2, \cdots$ it follows that $D=\bigcup_{r=1}^{\infty} D_{r}$.

Because $\sum_{j=1}^{\infty} d_{j}<\infty$ it follows that each set $D_{r}$ is finite and hence the sequence $\left\{d_{j}\right\}$ can be rearranged to give a sequence $\left\{d_{j_{n}}\right\}$ where $d_{j_{n}} \geqq d_{j_{n+1}}$. 
We define the sequence $\left\{c_{n}\right\}$ by letting $c_{n}=n d_{J_{n}}$. Now for each $p=1,2, \cdots$ we have

$$
\begin{aligned}
\sum_{n=1}^{p} d_{j_{n}} & <I_{1}(f) \\
\therefore p . d_{j_{p}} & <I_{1}(f) \\
\therefore c_{p} & <I_{1}(f)
\end{aligned}
$$

On the other hand if we take $\varepsilon>0$ we can choose $N$ such that for each $p=1,2, \cdots$ we have

$$
\begin{aligned}
& \sum_{n=N+1}^{N+p} d_{j_{n}}<\varepsilon \\
& \therefore p . d_{j_{N+p}}<\varepsilon \\
& \therefore c_{N+p}<\varepsilon\left(1+\frac{N}{p}\right) \\
& \therefore \lim _{p \rightarrow \infty} \sup c_{N+p} \leqq \varepsilon
\end{aligned}
$$

Since $\varepsilon$ is arbitrary it follows that $\left\{c_{n}\right\}$ has limit zero.

LEMMA 2.2. For each $\lambda>0$ we have

$$
I_{1}\left(f, F_{\lambda}\right) \leqq N \lambda+\sum_{n=N+1}^{\infty} d_{j_{n}}
$$

for all $N=1,2,3, \cdots$

ProOF. Since $F_{\lambda}$ is open

$$
\begin{aligned}
I_{1}\left(f, F_{\lambda}\right) & =\int_{F_{\lambda}}\left|f^{(1)}(x)\right| d x \\
& =\sum_{j=1}^{\infty} \int_{F_{\lambda \cap\left(a_{1 j}, b_{1 j}\right)}}\left|f^{(1)}(x)\right| d x \\
& =\sum_{j=1}^{\infty}\left|\int_{F_{\lambda \cap} \cap\left(a_{1 j}, b_{1 j}\right)} f^{(1)}(x) d x\right|
\end{aligned}
$$

Now suppose $d_{j} \geqq \lambda$ Consider the case where

$$
f\left(a_{1 j}\right) \leqq 0<\lambda \leqq f\left(b_{1 j}\right)
$$

We can choose $\left(\xi_{j}, \zeta_{j}\right)$ such that $\left(\xi_{j}, \zeta_{j}\right)=F_{\lambda} \cap\left(a_{1 j}, b_{1 j}\right)$ and also $f\left(\xi_{j}\right)=0$, $f\left(\zeta_{j}\right)=\lambda$. 


$$
\therefore \int_{F_{\lambda} \cap\left(a_{1 j}, b_{1 j}\right)} f^{(1)}(x) d x=\int_{\left(\xi_{j}, \zeta_{j}\right)} f^{(1)}(x) d x=\lambda .
$$

By using similar reasoning it can be shown in all cases where $d_{j} \geqq \lambda$ that

$$
\left|\int_{F_{\lambda} \cap\left(a_{1 j}, b_{1 j}\right)} f^{(1)}(x) d x\right| \leqq \lambda .
$$

Now if we choose $N_{\lambda}$ such that

$$
d_{j N_{\lambda}} \geqq \lambda>d_{j_{N_{\lambda}+1}}
$$

then we have

$$
\begin{aligned}
I_{1}\left(f, F_{\lambda}\right) & \leqq N_{\lambda} \cdot \lambda+\sum_{n=N_{\lambda}+1}^{\infty}\left|\int_{F_{\lambda} \cap\left(a_{1 j}, b_{1 j n}\right)} f^{(1)}(x) d x\right| \\
& \leqq N_{\lambda} \cdot \lambda+\sum_{n=N_{\lambda}+1}^{\infty}\left|\int_{\left(a_{1 j n}, h_{1 j n}\right)} f^{(1)}(x) d x\right| \\
& =N_{\lambda} \cdot \lambda+\sum_{n=N_{\lambda}+1}^{\infty} d_{j_{n}}
\end{aligned}
$$

and it is easily seen that for each $N=1,2, \cdots$

$$
N_{\lambda} \cdot \lambda+\sum_{n=N_{\lambda}+1}^{\infty} d_{j_{n}} \leqq N . \lambda+\sum_{n=N+1}^{\infty} d_{j_{n}}
$$

THEOREM $2.3 \lim _{\lambda \rightarrow 0} I_{1}\left(f, F_{\lambda}\right)=0$.

Proof. Let $\varepsilon>0$. Choose $N$ such that $\sum_{n=N+1}^{\infty} d_{j_{n}}<\varepsilon / 2$ and then choose $\lambda$ sufficiently small such that $N \lambda<\varepsilon / 2$. It follows that

$$
\begin{aligned}
I_{1}\left(f, F_{\lambda}\right) & \leqq N \lambda+\sum_{n=N+1}^{\infty} d_{j_{n}}<\varepsilon \\
\therefore \lim _{\lambda \rightarrow 0} I_{1}\left(f, F_{\lambda}\right) & =0 .
\end{aligned}
$$

\section{Variation in $S_{m+1}$}

Let $m$ be a natural number and suppose that $f \in S_{m+1}$. The $(m+1)$ th order variation of $f$ on an open set $U$ can be defined as

$$
I_{m+1}(f, U)=\int_{U}\left|f^{(m+1)}(x)\right| d x
$$


and in the case $U=R$ we have the total $(m+1)$ th order variation usually denoted by $I_{m+1}(f)$. Since $f^{(m+1)}$ is continuous and has compact support it follows from the definition that

$$
I_{m+1}(f)<\infty \text {. }
$$

Consider the intervals $\left(a_{1 j}, b_{1 j}\right)$. If we write $h_{1 j}=b_{1 j}-a_{1 j}$ and if we choose $L$ such that $\operatorname{spt}(f) \subset[-L / 2, L / 2]$ then

$$
\sum_{j=1}^{\infty} h_{1 j} \leqq L
$$

We define $a_{2 j} \in\left(a_{1 j}, b_{1 j}\right)$ such that $\left|f^{(1)}\left(a_{2 j}\right)\right| \geqq\left|f^{(1)}(x)\right|$ for all $x \in\left(a_{1 j}, b_{1 j}\right)$. Since $a_{2 j}$ is a local extremum of $f^{(1)}(x)$ it follows that $f^{(2)}\left(a_{2 j}\right)=0$.

Define $b_{2 j}=\inf \left\{x \mid x \geqq b_{1 j}\right.$ and $\left.f^{(2)}(x)=0\right\}$. Obviously $f^{(2)}\left(b_{2 j}\right)=0$ and we also know that if $b_{1 j} \leqq a_{1 k}$ then from the definition of $b_{2 j}$ we have $b_{2 j} \leqq a_{2 k}$. Hence the intervals $\left(a_{2 j}, b_{2 j}\right)$ are mutually disjoint. We also know that $b_{1 j} \in\left(a_{2 j}, b_{2 j}\right]$. If we write $h_{2 j}=b_{2 j}-a_{2 j}$ then we have

$$
\sum_{j=1}^{\infty} h_{2 j} \leqq L
$$

We can deduce in addition that

$$
\left|f^{(1)}\left(a_{2 j}\right)\right| \geqq \frac{\left|f\left(b_{1 j}\right)-f\left(a_{1 j}\right)\right|}{b_{1 j}-a_{1 j}}=\frac{d_{j}}{h_{1 j}}
$$

If $m=1$ we need not continue. Otherwise suppose that for some natural number $i$ with $2 \leqq i \leqq m$ we have mutually disjoint intervals $\left(a_{i j}, b_{i j}\right)$ with $f^{(i)}\left(a_{i j}\right)=f^{(i)}\left(b_{i j}\right)=0$ and with a point $b_{i-1 j} \in\left(a_{i j}, b_{i j}\right]$ such that $f^{(i-1)}\left(b_{i-1 j}\right)$ $=0$. Define $a_{i+1 j} \in\left(a_{i j}, b_{i j}\right)$ such that $\left|f^{()}\left(a_{i+1 j}\right)\right| \geqq\left|f^{(i)}(x)\right|$ for all $x \in\left(a_{i j}, b_{i j}\right)$. Since $a_{i+1 j}$ is a local extremum of $f^{(i)}(x)$ it follows that $f^{(i+1)}\left(a_{i+1 j}\right)=0$. We define $b_{i+1 j}=\inf \left\{x \mid x \geqq b_{i j}\right.$ and $\left.f^{(i+1)}(x)=0\right\}$. Obviously $f^{(i+1)}\left(b_{i+1 j}\right)=0$ and we also know that if $b_{i j} \leqq a_{i k}$ then from the definition of $b_{i+1 j}$ we have $b_{i+1 j} \leqq a_{i+1 k}$. Hence the intervals $\left(a_{i+1 j}, b_{i+1 j}\right)$ are mutually disjoint. We know that $b_{i j} \in\left(a_{i+1 j}, b_{i+1 j}\right]$ and if we write $h_{i+1 j}=b_{i+1 j}-a_{i+1 j}$ then it is true that

We also have

$$
\sum_{j=1}^{\infty} h_{i+1 j} \leqq L
$$

$$
\left|f^{(i)}\left(a_{i+1 j}\right)\right| \geqq \frac{\left|f^{(i-1)}\left(b_{i-1 j}\right)-f^{(i-1)}\left(a_{i j}\right)\right|}{b_{i-1 j}-a_{i j}}
$$

and since $f^{(i-1)}\left(b_{i-1 j}\right)=0$ and $h_{i j}=b_{i j}-a_{i j} \geqq b_{i-1 j}-a_{i j}$ it follows that 


$$
\left|f^{(i)}\left(a_{i+1 j}\right)\right| \geqq \frac{\left|f^{(i-1)}\left(a_{i j}\right)\right|}{h_{i j}}
$$

By induction it follows that the intervals $\left(a_{i+1 j}, b_{i+1 j}\right)$ can be defined for each $i=2,3, \cdots, m$. Now since the intervals $\left(a_{m j}, a_{m+1 j}\right)$ are disjoint it follows that

$$
\begin{gathered}
I_{m+1}(f)=I_{1}\left(f^{(m)}\right) \geqq \sum_{j=1}^{\infty}\left|f^{(m)}\left(a_{m+1 j}\right)-f^{(m)}\left(a_{m j}\right)\right| \\
\therefore I_{m+1}(f) \geqq \sum_{j=1}^{\infty}\left|f^{(m)}\left(a_{m+1 j}\right)\right|
\end{gathered}
$$

and by repeated use of (2) and finally using (1) this becomes

$$
I_{m+1}(f) \geqq \sum_{j=1}^{\infty} \frac{d_{j}}{h_{m j} h_{m-1 j} \cdots \cdot h_{2 j} h_{1 j}}
$$

It is convenient now to quote a standard inequality

Lemma 3.1. Let $x_{i j}>0$ each $i=1, \cdots, m$ and each $j=1, \cdots, p$. Suppose that $\sum_{j=1}^{p} x_{i j} \leqq L$ for each $i=1, \cdots, m$. Then

$$
\sum_{j=1}^{p} \frac{1}{x_{m j} x_{m-1 j} \cdots \cdot x_{2 j} x_{1 j}} \geqq \frac{p^{m+1}}{L^{m}}
$$

LEMMA 3.2. The sequence $\left\{d_{j}\right\}$ can be rearranged to a sequence $\left\{d_{j_{n}}\right\}$ such that $d_{j_{n}} \geqq d_{j_{n+1}}$ for all $n=1,2, \cdots$ and in fact we can write

$$
d_{j_{n}}=\frac{k_{n}}{n^{m+1}} \text { all } n=1,2, \cdots
$$

where $\left\{k_{n}\right\}$ is bounded and has limit zero.

PROOF. Define $k_{n}=n^{m+1} d_{j_{n}}$. Now for each $p=1,2, \cdots$ we have

$$
\begin{aligned}
\sum_{n=1}^{p} \frac{d_{j_{n}}}{h_{m j_{n}} h_{m-1 j_{n}} \cdots h_{2 j_{n}} h_{1 j_{n}}} & \leqq I_{m+1}(f) \\
\therefore d_{j_{p}}\left(\sum_{n=1}^{p} \frac{1}{h_{m j_{n}} \cdots \cdot h_{2 j_{n}} h_{1 j_{n}}}\right) & \leqq I_{m+1}(f) \\
\therefore \frac{k_{p}}{p^{m+1}} \cdot \frac{p^{m+1}}{L^{m}} & \leqq I_{m+1}(f) \\
\therefore k_{p} & \leqq L^{m} I_{m+1}(f)
\end{aligned}
$$


On the other hand if we take $\varepsilon>0$ we can choose $N$ such that for each $p=1,2, \cdots$ we have

$$
\sum_{n=N+1}^{N+p} \frac{d_{j_{n}}}{h_{m j_{n}} \cdots \cdot h_{2 j_{n}} h_{1 j_{n}}}<\frac{\varepsilon}{L^{m}}
$$

Thus as above we deduce that

$$
\begin{aligned}
& \frac{k_{N+p}}{(N+p)^{m+1}} \frac{p^{m+1}}{L^{m}}<\frac{\varepsilon}{L^{m}} \\
& \therefore k_{N+p}<\varepsilon\left(1+\frac{N}{p}\right)^{m+1}
\end{aligned}
$$

$$
\therefore \limsup _{p \rightarrow \infty} k_{N+p} \leqq \varepsilon
$$

Since $\varepsilon$ is arbitrary it follows that $\left\{k_{n}\right\}$ has limit zero.

THEOREM 3.3 For each $\lambda$ with $0<\lambda<1$ we have

$$
\lambda^{1 /(m+1)-1} I_{1}\left(f, F_{\lambda}\right) \leqq 1+\frac{(2 L)^{m} I_{m+1}(f)}{m}
$$

and in addition

$$
\lim _{\lambda \rightarrow 0} \lambda^{1 /(m+1)-1} I_{1}\left(f, F_{\lambda}\right)=0
$$

Proof. Choose $N$ such that $1 / 2 N \leqq \lambda^{1 /(m+1)}<1 / N$

$$
\begin{aligned}
I_{1}\left(f, F_{\lambda}\right) & \leqq N \lambda+\sum_{n=N+1}^{\infty} d_{j_{n}} \\
& \leqq \lambda^{m /(m+1)}+\sum_{n=N+1}^{\infty} \frac{L^{m} \cdot I_{m+1}(f)}{n^{m+1}} \\
& \leqq \lambda^{m /(m+1)}+L^{m} \cdot I_{m+1}(f) \cdot \int_{N} \frac{d x}{x^{m+1}} \\
& =\lambda^{m /(m+1)}+\frac{L^{m} \cdot I_{m+1}(f)}{m N^{m}} \\
& \leqq\left\{1+\frac{(2 L)^{m} \cdot I_{m+1}(f)}{m}\right\} \cdot \lambda^{m /(m+i}
\end{aligned}
$$


Now choose $\varepsilon>0$, and find $N$ such that

$$
k_{n} \leqq 2 m\left(\frac{\varepsilon}{4}\right)^{m+1} \text { all } n \geqq N
$$

Take $\lambda$ sufficiently small that $0<\lambda^{1 /(m+1)}<\varepsilon / 2 N$. Choose $N_{1} \geqq N$ with

$$
\frac{\varepsilon}{4 N_{1}} \leqq \lambda^{1 /(m+1)}<\frac{\varepsilon}{2 N_{1}} .
$$

Now it follows that

$$
\begin{aligned}
I_{1}\left(f, F_{\lambda}\right) & \leqq N_{1} \lambda+\sum_{n=N_{1}+1}^{\infty} d_{j_{n}} \\
& \leqq \frac{\varepsilon}{2} \cdot \lambda^{m /(m+1)}+\sum_{n=N_{1}+1}^{\infty} \frac{2 m \cdot(\varepsilon / 4)^{m+1}}{n^{m+1}} \\
& \leqq \frac{\varepsilon}{2} \cdot \lambda^{m /(m+1)}+2 m\left(\frac{\varepsilon}{4}\right)^{m+1} \int_{N_{1}}^{\infty} \frac{d x}{x^{m+1}} \\
& =\frac{\varepsilon}{2} \cdot \lambda^{m /(m+1)}+2 m\left(\frac{\varepsilon}{4}\right)^{m+1} \frac{1}{m N_{1}^{m}} \\
& \leqq \varepsilon \lambda^{m /(m+1)}
\end{aligned}
$$

Since $\varepsilon$ was arbitrarily chosen it follows that

$$
\lim _{\lambda \rightarrow 0} \lambda^{1 /(m+1)-1} I_{1}\left(f, F_{\lambda}\right)=0
$$

\section{References}

[1] W. H. Fleming and R. Rishell, 'An integral formula for total gradient variation', Arch. Math. 11 (1960) 218-222.

[2] C. Goffman, 'Lower semi-continuity and area functionals. The non-parametric case, Rend. Circ. Mat. Palermo (2), 2 (1953) 203-235.

[3] C. Goffman, 'Approximation of non-parametric surfaces of finite area', J. Math. and Mech. 12, 5 (1963), 737-746.

[4] P. G. Howlett, 'Approximation to summable functions' (Thesis, University of Adelaide (1971) 33-148).

[5] J. H. Michael, 'The equivalence of two cases for non-parametric discontinuous surfaces', Illinois J. Math. 7 (1963), 59-78. 
[6] J. H. Michael, 'Approximation of functions by means of Lipschitz functions', J. Austral. Math. Soc. 3, 2 (1963), 134-150.

[7] J. H. Michael, 'Lipschitz approximations to summable functions', .4cth Math. 3 (1964), 73-95.

School of Mathematics

South Australian Institute of Technology

Adelaide, South Australia 